\title{
Simulación numérica de la interconexión de corte de vigas mixtas de acero de alta resistencia y hormigón UHPC
}

\section{Numerical simulation of the interconnection of high strength steel composite beams and UHPC concrete}

Fecha de entrega: 27 de agosto 2011 Fecha de aceptación: 18 de noviembre 2011

\section{Andrés Panés}

ITASCA S.A., Dr. José Luis Aguilar 1178, Providencia, Santiago, Chile, andres.panes@itasca.cl (anteriormente en el Instituto de Estructuras de Hormigón de la Universidad RWTH-Aachen, Alemania)

Este artículo presenta análisis numéricos de vigas mixtas de acero de alta resistencia y hormigón de ultra alto desempeño UHPC. Debido a la alta resistencia a la compresión del hormigón UHPC, son posibles estructuras muy esbeltas con elementos de hormigón delgados. Sin embargo, tensiones locales aumentan considerablemente en las uniones entre estos dos materiales debido a la mayor capacidad de carga. Para ello, han sido desarrollados conectores de corte continuos. Simulaciones numéricas son explicadas para estudiar vigas mixtas con conectores de corte (resortes). También se presentan detalles de los hormigones UHPC. Se modela una viga mixta con propiedades no lineales de los materiales. Ensayos de flexión de vigas son simulados con ABAQUS para calibrar el modelo numérico. Los resultados numéricos concuerdan relativamente bien con los resultados experimentales incluso después de iniciada la primera grieta. Además se implementó un modelo tridimensional con elementos de volumen para las partes de acero y hormigón y elementos de resorte no lineales para la conexión de corte. Los resultados de ensayos pushout fueron usados en el modelo no lineal de los elementos de resorte. La aplicación de cargas puntuales en el modelo debido a los resortes explican las tensiones máximas y debido a esto la pérdida de rigidez más temprana comparada con los ensayos de la viga. La carga última y la ocurrencia de deslizamiento fueron estimadas satisfactoriamente.

Palabras clave: vigas mixtas, UHPC, conectores de corte continuos, simulaciones numéricas ABAQUS 3D, leyes no lineales del hormigón
This article presents numerical analyses on composite beams made of high strength steel and Ultra High Performance Concrete (UHPC). Due to the high compressive strength of UHPC very slender structures with thin concrete elements are feasible. However, local stresses in the composite joints increase significantly due to the higher load capacity. For that, continuous shear connectors have been developed. Numerical simulations are explained to the study of composite beams related to the shear connection (springs). Details of UHPC are also presented. A composite beam with non-linear material properties was modelled. Beam-bending tests were simulated with ABAQUS to calibrate the numerical model. The numerical results agree relatively well with the test results, even after first crack initiation. In addition, a three dimensional model with volume elements for the steel and the concrete parts and non-linear spring elements for the shear connection were implemented. The results from performed push-out tests were used in the nonlinear model of the springs elements. The punctual application of the forces in the model due to the springs were the reason for tension peaks and on this account there was an earlier loss of stiffness compared to the beam test result. The ultimate load and the occurring slip were satisfactorily calculated.

Keywords: composite beams, UHPC, continuous shear connectors, ABAQUS $3 D$ numerical simulations, concrete non linear law

simulación numérica y la modelación física para calibrar y validar el modelo numérico. La determinación de las propiedades y respuesta estructural de los materiales a través de ensayos de modelos experimentales ha permitido la calibración de los modelos numéricos. 
El primer objetivo de este trabajo es simular numéricamente la interconexión a corte de vigas mixtas de acero de alta resistencia y hormigón UHPC (Ultra High Performance Concrete) utilizando el programa computacionalABAQUS. ABAQUS/CAE es una herramienta multipropósito que se basa en el Método de Elementos Finitos MEF para resolver diversas aplicaciones en el campo de la ingeniería, incluidos problemas de tipo no lineales. Los tipos de interconexión utilizados fueron rígidos y flexibles (elementos de resorte). Otra posibilidad pueden ser elementos de interfaz que no forman parte de este trabajo.

El segundo objetivo es comparar los resultados de los cálculos numéricos obtenidos de las simulaciones con ABAQUS con los resultados de los ensayos de laboratorio obtenidos por Kaufmann (2010), con el fin de realizar una modelación numérica válida.

Este trabajo se encuentra inmerso dentro de un programa de investigación sobre hormigones de ultra alto rendimiento UHPC y aceros de alta resistencia que se lleva a cabo en el Instituto de Estructuras de Hormigón (IMB) de la Universidad RWTH-Aachen, Alemania.

\section{Estudio de vigas mixtas}

Las vigas mixtas (composite beams) son elementos estructurales de alta resistencia a la compresión. Esto permite la construcción de estructuras más esbeltas con elementos más delgados. Sin embargo, se desarrollan altas tensiones locales en las conexiones debido a que estos elementos son capaces de soportar grandes cargas. Para ello se han diseñado conectores de corte continuos (Rauscher et al. 2011).

El trabajo de Aribert y Labib (1982) surge como un primer paso hacia la aplicación del MEF para el cálculo de vigas mixtas. Proporciona un método de cálculo eficaz para modelar el comportamiento estructural no lineal de vigas mixtas. Aribert y Labib (1982) describen en su trabajo un modelo llamado "bastón" el cual es hasta ahora el más sencillo y más eficiente modelo de elementos finitos para el cálculo de vigas mixtas (Figura 1).

En este modelo se idealiza la unión entre la losa y la viga de acero con un resorte con resistencia a flexión infinita, el cual provee la transferencia de las fuerzas de corte.

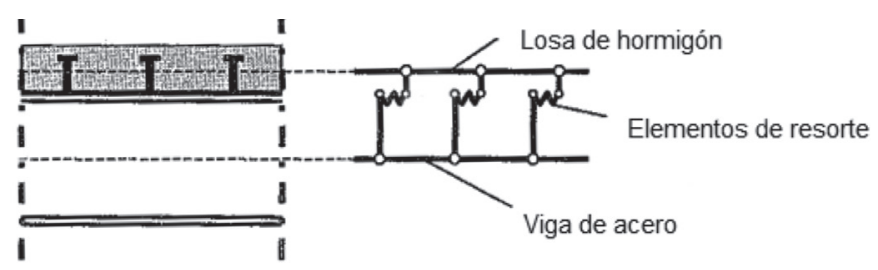

Figura1: Esquema del modelo "bastón” (Aribert y Labib 1982)

La función de los elementos de resorte horizontales es unir la losa de hormigón y la viga de acero con el fin de poder considerar el desplazamiento relativo entre ellos. Se debe tener presente que es necesario determinar la curva característica de fuerza-deformación del resorte para poder simular la zona de carga, la cual se obtiene a partir de un ensayo push-out. El ensayo push-out realizado en vigas compuestas consiste en aplicar gradualmente una fuerza de tracción para separar el bloque de hormigón y la viga de acero por deslizamiento longitudinal. Por lo tanto a partir de ensayos push-out se pueden obtener gráficas de fuerza aplicada versus deslizamiento relativo entre el acero y el hormigón, los cuales resultan de mucha utilidad para implementar modelos numéricos (Figura 2).

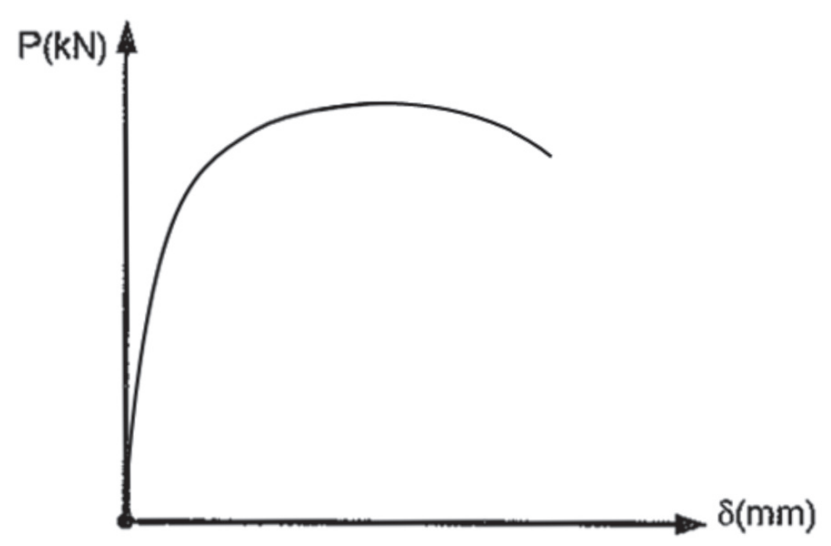

Figura 2: Curva característica de fuerza versus deslizamiento en el ensayo push-out

\section{Simulación de la interconexión}

Las fuerzas de corte horizontales se transmiten a través de la conexión entre la viga de acero y losa de hormigón. Para esto es importante que la unión tenga la rigidez y resistencia adecuada, además de tener la ductilidad suficiente. De esta manera, la viga de acero y la losa de hormigón armado conectados entre sí, se comportarán como una viga mixta. 
En consecuencia la modelación de vigas mixtas busca simular el efecto de corte longitudinal entre la viga de acero y la losa de hormigón.

Actualmente con la existencia de modelos de análisis basados en el MEF es posible reproducir comportamientos estructurales complejos. La utilización de dichos modelos permite realizar estudios preliminares previos a la realización de ensayos también complejos para analizar el problema a investigar y conocer los parámetros que van a gobernar dicho problema. Por ejemplo se pueden obtener de forma aproximada las cargas máximas, tensiones y deformaciones y así poder diseñar y preparar los ensayos más adecuados.

\section{Hormigón UHPC}

El hormigón UHPC está basado en el principio de minimizar defectos como las microfisuras y los vacíos, logrando alcanzar un mayor porcentaje de la carga última potencial e incrementando sustancialmente la durabilidad. Este hormigón posee una baja relación agua/cemento, permitiendo tener una alta resistencia a la compresión. Al generar una mezcla mucho más densa a través de la eliminación de los agregados de mayor tamaño y la optimización de la masa granular, se obtiene una matriz con una alta resistencia a la compresión por encima de los $180 \mathrm{MPa}$. Si a esto se agrega la aplicación de un tratamiento de curado mediante calor, se obtiene una mejora de la microestructura del material e incremento de la resistencia a la compresión por sobre los $200 \mathrm{MPa}$.

La porosidad baja del UHPC, entre 1 y $2 \%$, lo hace casi impermeable. Por ello tiene una gran capacidad impermeabilizante y también soporta la acción de productos químicos. Estos hormigones se consideran los hormigones del futuro, puesto que permiten diseñar secciones más esbeltas, edificios más altos, puentes más largos y estructuras más durables. Es uno de los últimos grandes avances del sector de la construcción, lo cual permite crear estructuras de grandes luces, espesores reducidos y elevadas alturas.

\section{Descripción y análisis de los ensayos}

Para ensayar la viga se ha empleado la mitad de un perfil HEM 900 con un corte en forma de rompecabezas en la parte superior del alma como se muestra en la Figura 3. La sección rompecabezas de la viga de acero fue incrustada en $90 \mathrm{~cm}$ de ancho y $10 \mathrm{~cm}$ de espesor de hormigón (ver Figuras 4 y 5).

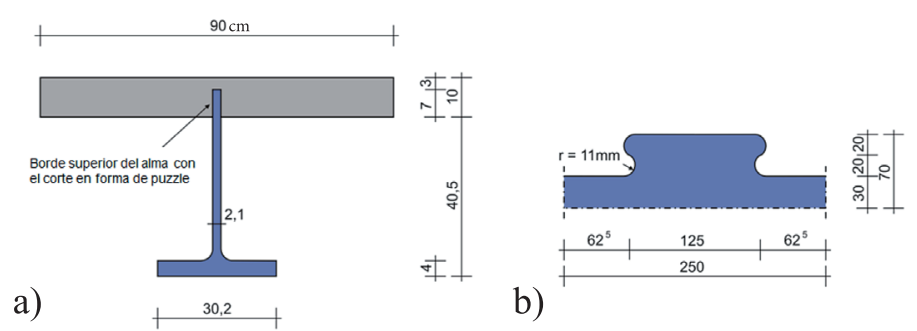

Figura 3: a) Sección transversal de la viga ensayada HEM900 H4 y b) geometría del rompecabezas (Kaufmann 2010)

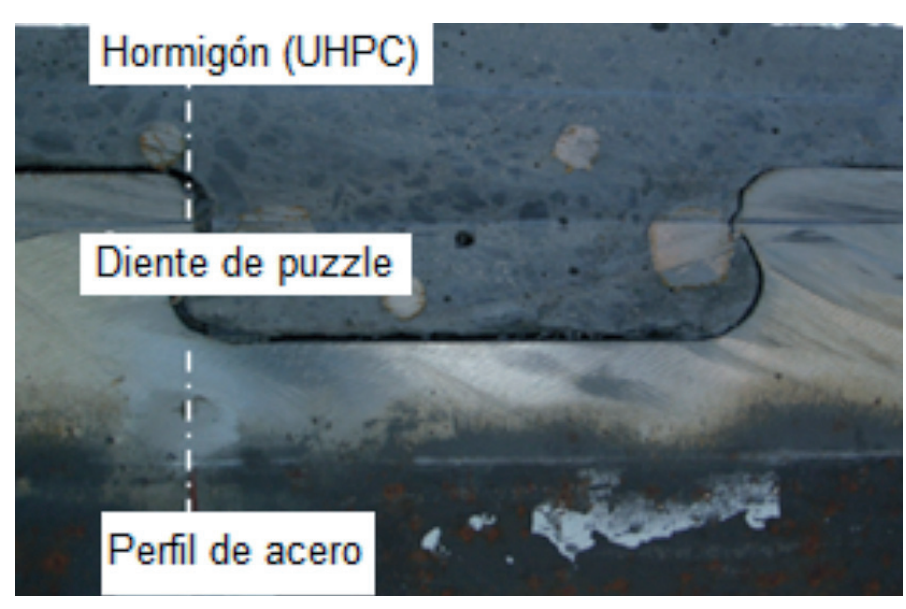

Figura 4: Unión entre el acero y el hormigón (Kaufmann 2010)

\section{Ensayo push-out}

A partir de ensayos push-out se puede obtener la curva característica de fuerza aplicada versus deslizamiento relativo entre la losa de hormigón y la viga de acero. El ensayo push-out consiste en aplicar una carga axial sobre el perfil de acero hasta la falla de la viga mixta, de manera que exista transferencia de esfuerzos directamente a los conectores (ver Figura 6). Para reproducir y así poder comparar los resultados de los ensayos push-out se ha utilizado el procedimiento experimental según el EC4 (Eurocódigo 4).

\section{Resultado del ensayo push-out}

La Figura 7 muestra la relación de fuerza-deslizamiento a partir del ensayo push-out. El ensayo presentó un rango lineal-elástico hasta aproximadamente los $400 \mathrm{kN}$ por 
conector (resorte). A continuación en su estado no lineal, la carga se incrementó hasta llegar a su carga máxima de $750 \mathrm{kN}$ con un deslizamiento de $9 \mathrm{~mm}$

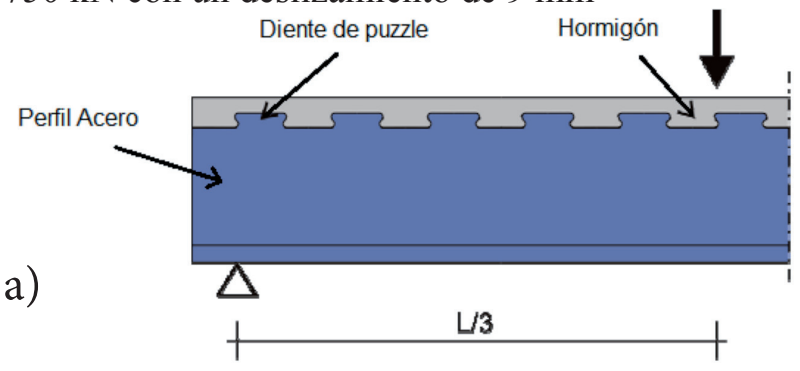

b)

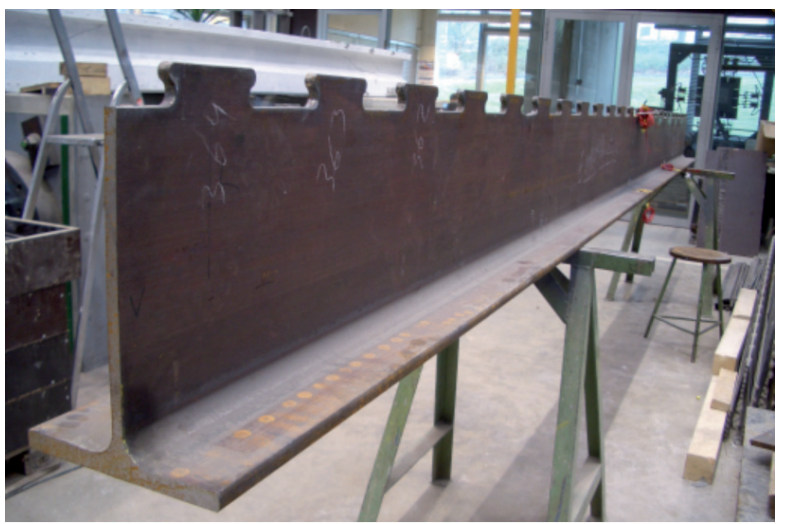

c)

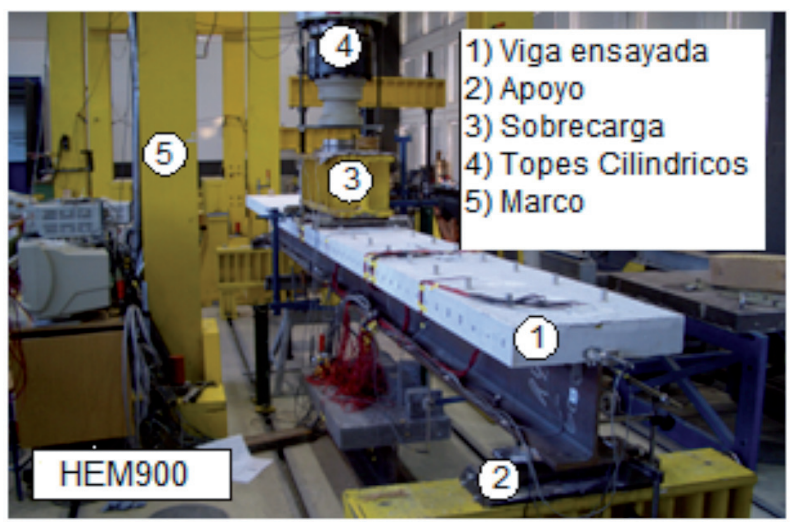

Figura 5: a) Idealización de la interconexión, b) perfil longitudinal de la viga de acero y c) viga a ensayar (Kaufmann 2010) a)

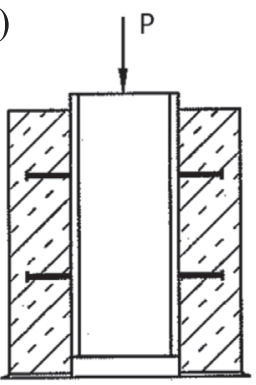

b)

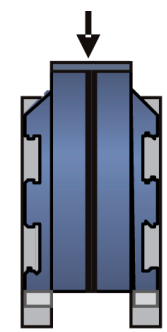

Vista frontal

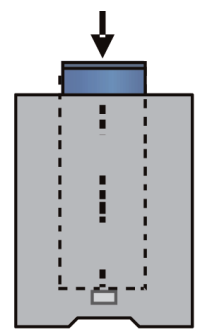

Vista lateral Vista en planta
Figura 6: Ensayo push-out a) esquema EC4 y b) esquema de vistas de los ensayos de laboratorio

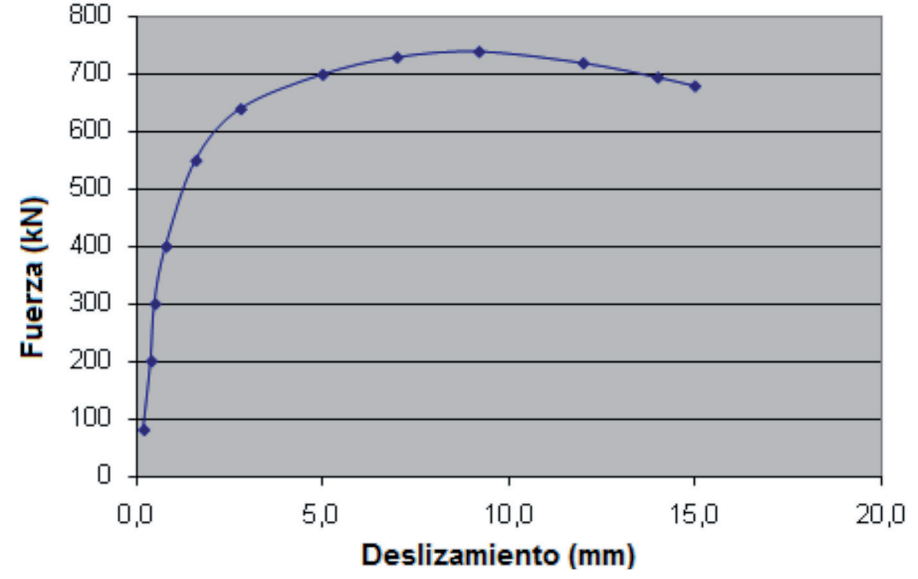

Figura 7: Curva característica del rompecabezas, ensayo pushout (Kaufmann 2010)

\section{Ensayo a flexión}

Se realizó un ensayo a escala de la viga mixta a estudiar. La viga denominada HEM900, es la viga patrón o de referencia con la cual más adelante se compararán los resultados experimentales con los resultados de la simulación realizada mediante el programa ABAQUS (2009). El método utilizado fue el de "4 puntos", el cual es requerido para medir el comportamiento de los materiales sometidos a cargas sobre una viga. Uno de los objetivos de este ensayo es determinar la curva momento-deflexión del prototipo. La viga mixta es soportada por dos apoyos como una viga simple y se aplica la carga en dos puntos. Los resultados se trazan en un diagrama esfuerzo-deformación y el esfuerzo máximo en el punto de ruptura es la resistencia a la flexión.

Cabe señalar que se realizaron 2 ensayos y los resultados fueron idénticos. Se realizó el ensayo a flexión de "4 puntos" en una viga de acero de longitud $6 \mathrm{~m}$ y la distancia de los apoyos al borde fue de 0,125 m. Los dos puntos de carga se distribuyeron en el centro de la viga a una distancia de 1,0 m entre ellos (Figura 8).
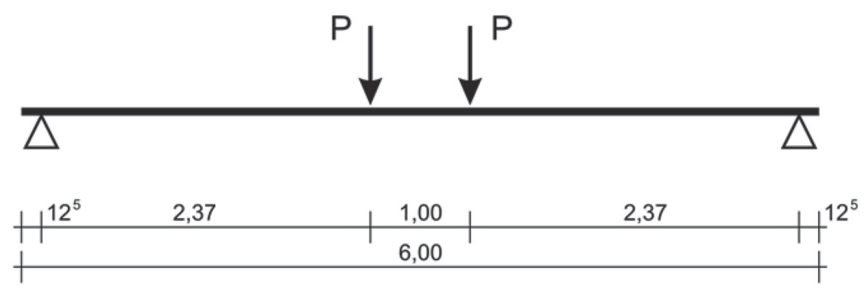

Figura 8: Sistema estático HEM900 
Los ensayos se realizaron con la máquina de ensayo servo hidráulica HB 5000 de la empresa Zwick/Roel. La carga del aparato se distribuyó a través de un travesaño con puntos de carga, tal como se muestra en la Figura 9.

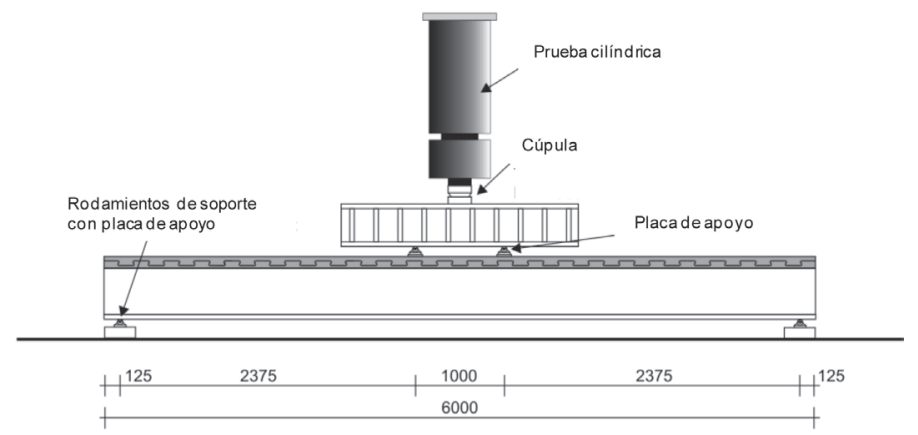

Figura 9: Montaje del ensayo a flexión de la viga HEM900

\section{Simulación con ABAQUS}

Con el fin de tener una herramienta numérica capaz de predecir adecuadamente el comportamiento de la viga mixta HEM900, se analizó la viga ensayada experimentalmente con el programa computacional ABAQUS. Se realizó una representación 3D de la viga mixta y se analizó el comportamiento de la interconexión entre la losa de hormigón y la viga de acero para luego comparar los resultados de la simulación con los resultados obtenidos experimentalmente por Kaufmann (2010).

Por razones de simetría y tamaño del modelo, se decidió modelar sólo un cuarto de la viga (Figura 10). Esto se pudo realizar, ya que la geometría, las condiciones de borde y las cargas presentan una doble simetría respecto a la sección transversal y al plano vertical longitudinal de la viga.

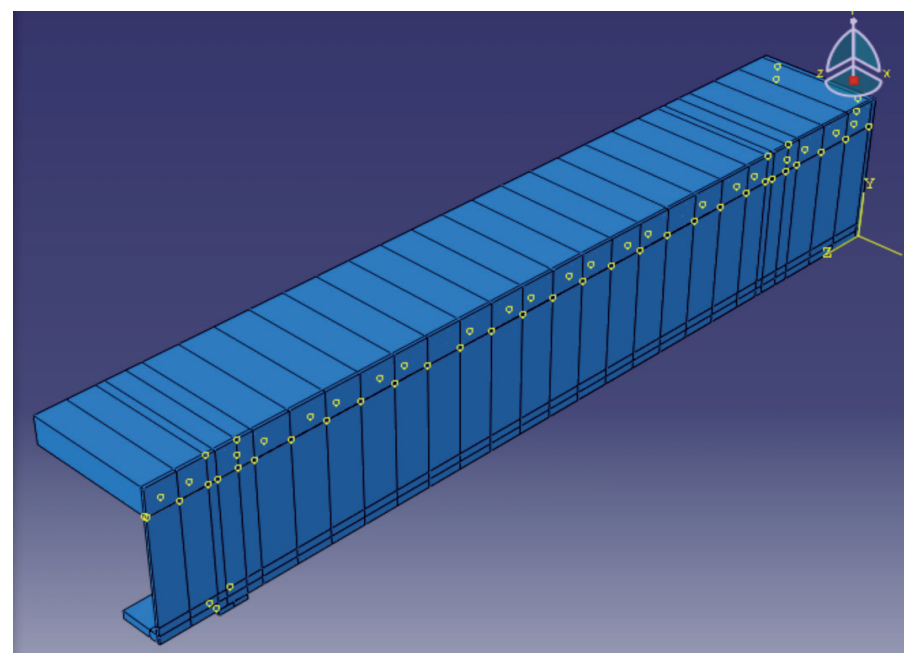

Figura 10: Un cuarto de la viga analizada

\section{Modelación de los materiales}

Para modelar el comportamiento del acero se ha empleado un modelo elasto-plástico usando la teoría de falla de Von Mises. Esta teoría de rotura también se llama teoría de la energía cortante o teoría de Von Mises y se emplea sólo para definir el principio de la fluencia.

En la modelación de estructuras mixtas con el MEF, la mayor dificultad se encuentra en la caracterización del hormigón armado. Para esto se empleó un modelo de daño plástico, ya que tiene una mejor aproximación a la curva real de comportamiento determinada a partir de estudios experimentales (Lee y Fenves, 1998).

\section{Modelo de daño plástico del hormigón}

El modelo de daño plástico del hormigón está basado en la suposición de daño isotrópico para aplicaciones en las cuales el hormigón está sujeto a condiciones de cargas arbitrarias, incluyendo cargas cíclicas. El modelo considera la degradación de la rigidez causada por la deformación plástica tanto en tensión como en compresión. Además permite representar el efecto de recuperación de rigidez frente a cargas cíclicas.

Los modelos de plasticidad y daño para el hormigón y materiales con características similares presentan las siguientes aplicaciones:

- Permite modelar el hormigón para todos los tipos de elementos estructurales: vigas, barras y sólidos.

- Usa los conceptos de elasticidad isotrópica en combinación con plasticidad isotrópica a tensión y compresión para representar el comportamiento elástico del hormigón.

- Puede ser usado en hormigón sin refuerzo como parte de un análisis primario para aplicaciones de estructuras con hormigón armado.

- Requiere la definición de la parte elástica del material.

Para la calibración del modelo de daño plástico se deben introducir las curvas de comportamiento para la compresión y la tracción del hormigón como puntos discretos $(\sigma-\varepsilon)$, tomadas a partir de ensayos uniaxiales (ver Figura 11). 


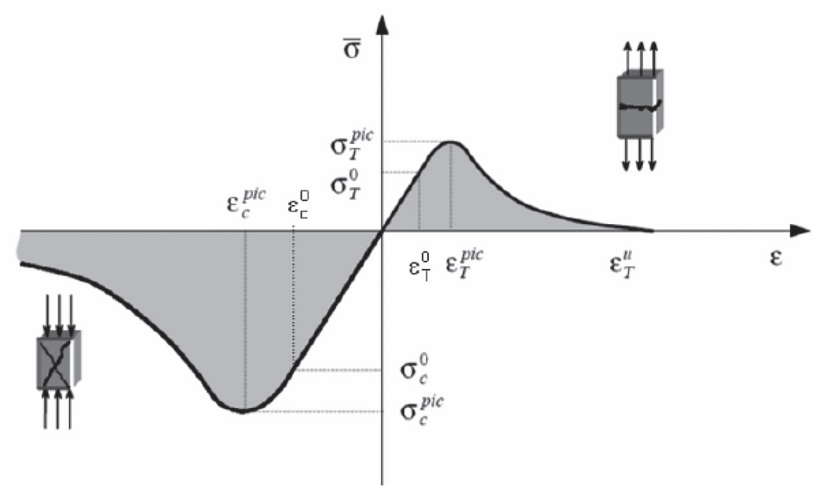

Figura 11: Comportamiento bajo compresión y tracción (ensayo uniaxial)

Con el fin de simular el comportamiento elástico del hormigón se debe especificar el módulo de elasticidad y el coeficiente de Poisson. Para simular el daño plástico se deben introducir los resultados de la simulación del ensayo a flexión (4 puntos), los cuales son: concrete compression hardening y concrete tension stiffening. El primero describe el comportamiento no lineal del hormigón a compresión y el segundo define el comportamiento del hormigón en la zona de tensión.

\section{Resistencia a la flexo-tracción}

Para obtener la resistencia a la flexo-tracción se realizó el ensayo de flexión de 4 puntos. Para el ensayo se necesita una probeta en forma de barra con sección rectangular o circular. La tensión $\sigma$ se calcula a partir de los siguientes parámetros: momento flector $M$ y módulo resistente de la sección transversal $z$, según la expresión:

$$
\sigma=\frac{M}{z}
$$

En este ensayo la tensión máxima o tensión a la fractura corresponde a la resistencia a la flexión o módulo de rotura. La medida de la resistencia a la flexión se puede obtener a partir de dos tipos de ensayos. El ensayo de tres puntos y el ensayo de cuatro puntos. En nuestro caso se utilizó este último (ver Figura 12).

Kaufmann (2010) ensayó una serie de seis muestras rectangulares y para cada una obtuvo una curva característica. Estas curvas experimentales se usaron para calibrar el modelo realizado en ABAQUS del material UHPC sometido a tracción. Para la calibración se introdujeron los siguientes parámetros en el modelo: resistencia a compresión y a tracción, elasticidad y energía de falla. Para hacer la calibración del modelo del material UHPC es necesario modelar la curva flexotraccióndeflexión. Para esto se varió el valor de la energía de rotura entre 1 a $10 \mathrm{~N} / \mathrm{mm}$ y la resistencia a la tracción entre 7 a $12 \mathrm{~N} / \mathrm{mm}^{2}$, dichos intervalos son característicos para el UHPC. Cabe mencionar que se realizaron varios ensayos numéricos antes de definir la curva final. Esta curva debe estar dentro del rango de las 6 curvas obtenidas de los ensayos de laboratorio por Kaufmann (2010).
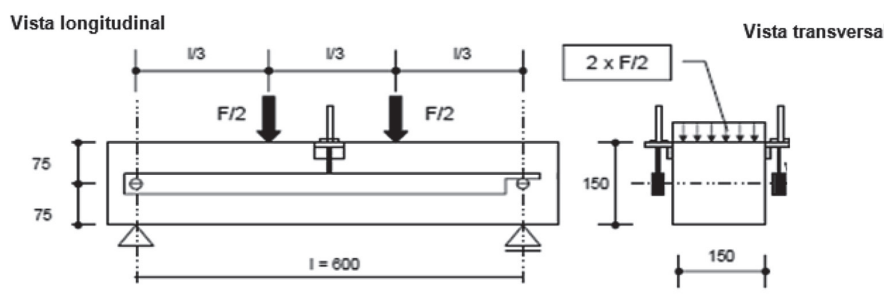

Figura 12: Ensayo de 4 puntos, dimensiones en mm

Finalmente, se eligió la curva con una energía de rotura de $2.8 \mathrm{~N} / \mathrm{mm}$ y una resistencia a la tracción de $10 \mathrm{~N} / \mathrm{mm}^{2}$, ya que esta curva comparada con los 6 ensayos experimentales (E1, E2,...E6) muestra una buena aproximación. Se puede apreciar que la curva simulada está contenida dentro de las curvas experimentales, por ende dichos parámetros (energía de rotura y resistencia a tracción) pueden ser utilizados. La Figura 13 muestra que el punto más alto de tensión, resistencia a flexo-tracción, es $20.53 \mathrm{~N} / \mathrm{mm}^{2}$ con una deflexión de $0.55 \mathrm{~mm}$.

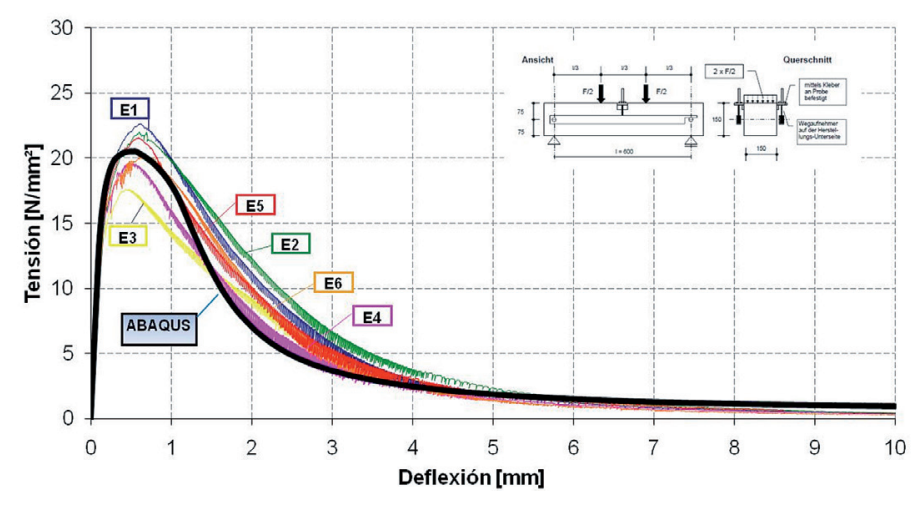

Figura 13: Tensión versus deflexión

La Figura 14 muestra las curvas de fuerza versus deflexión obtenidas del ensayo realizado en el laboratorio por Kaufmann (2010), junto a la curva de la simulación en ABAQUS. 


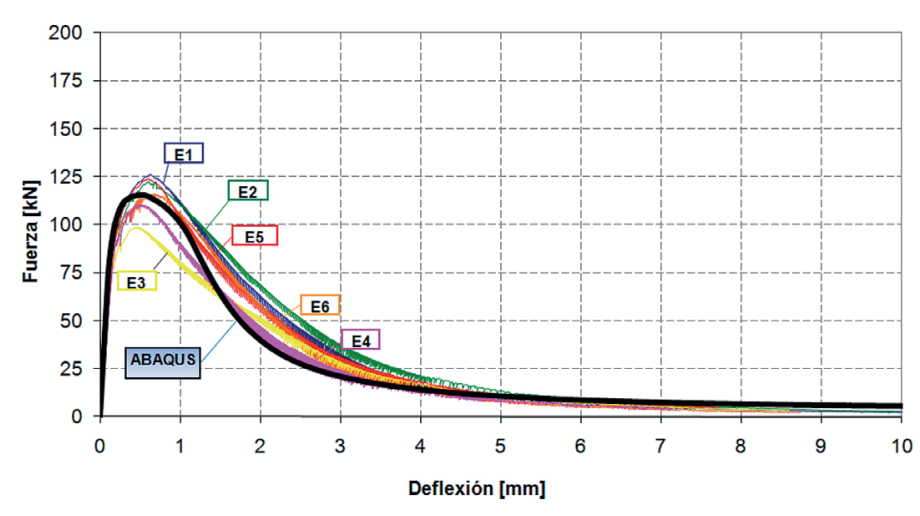

Figura 14: Fuerza versus deflexión

La Tabla 1 presenta los parámetros utilizados en la simulación. Los valores del ángulo de dilatación, excentricidad y coeficiente de balasto son parámetros característicos del hormigón UHPC y han sido utilizados en investigaciones numéricas con ABAQUS (Teworte, 2008).

Tabla 1: Parámetros del hormigón utilizados en la simulación

\begin{tabular}{|l|c|c|}
\hline & Unidad & Valor \\
\hline Módulo de elasticidad E & $\mathrm{N} / \mathrm{mm}^{2}$ & 60000 \\
\hline Resistencia cúbica a la compresión $\phi_{\mathrm{c}}$ & $\mathrm{N} / \mathrm{mm}^{2}$ & 180 \\
\hline Resistencia a la tracción $\phi_{\mathrm{t}}$ & $\mathrm{N} / \mathrm{mm}^{2}$ & 10 \\
\hline Energía de falla $\mathrm{G}_{\mathrm{f}}$ & $\mathrm{N} / \mathrm{mm}$ & 2.80 \\
\hline Ángulo de dilatación $\psi$ & $\circ$ & 35 \\
\hline Excentricidad $e$ & - & 0.1 \\
\hline Coeficiente de forma $\mathrm{K}_{\mathrm{c}}$ & - & 0.66 \\
\hline
\end{tabular}

\section{Módulo "Interacción"}

Se usa el módulo "Interacción" para definir el contacto entre dos regiones del modelo. El primer paso es crear las superficies que serán incluidas en las interacciones. No siempre es necesario crear las superficies con anticipación; si el modelo es simple o las superficies fáciles de seleccionar, se pueden indicar las superficies maestras (master) y esclavas (slave) directamente en la ventana cuando se crean las interacciones. Sin embargo, en este ejemplo, es más fácil definir las superficies separadamente y luego referirse a los nombres de esas superficies cuando se crean las interacciones.

La diferencia sustancial entre las dos superficies reside en que la superficie maestra puede penetrar en la esclava, pero no a la inversa. Por lo que la definición de éstas es importante, ya que pueden aparecer problemas de convergencia, penetración de unas superficies en otras y por ende distorsión en los resultados. Para definir estas superficies de una forma correcta, se debe tener en cuenta la rigidez y el mallado de las partes que integran el modelo. La superficie maestra debe ser la de mayor rigidez y, en el caso de materiales idénticos, la de mallado más grueso.

Debido al fenómeno principal que se requiere reproducir, que es el deslizamiento relativo entre dos superficies, se opta por modelar la viga de acero como un sólido homogéneo. De esta forma se garantiza la posibilidad de mallar de forma idéntica la viga y la losa de hormigón en su superficie de contacto y hacer coincidir uno a uno los nodos master con los nodos slave de la superficie vecina. En el caso de las barras de armadura interaccionan con la losa de hormigón por medio de la opción embeded element. Esta técnica elimina los grados de libertad perpendiculares al eje del elemento empotrado (Figura 15).

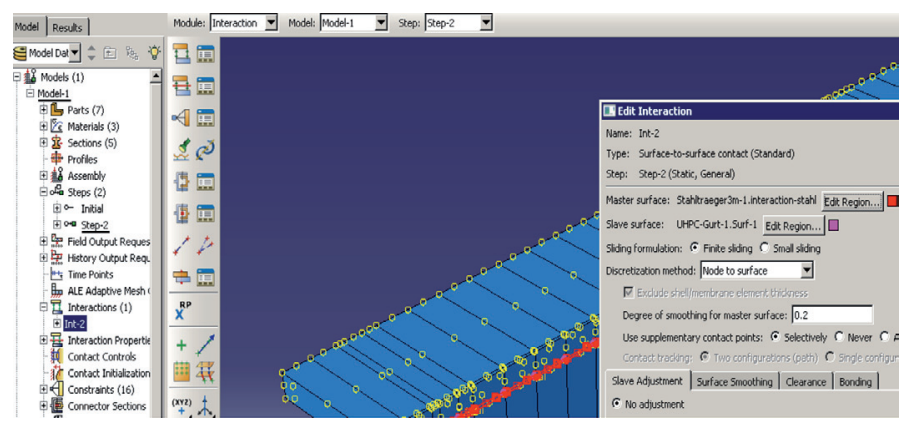

Figura 15: Interacción entre la viga de acero y la losa de hormigón UHPC

\section{Elementos de resorte}

Con el fin de simular la interconexión al corte entre el hormigón y el acero se utilizaron elementos de resorte. La base del MEF es la representación de un cuerpo mediante subdivisiones llamadas elementos. Las deformaciones y las fuerzas externas se relacionan entre sí mediante la rigidez y las relaciones constitutivas del elemento. Trabajando en régimen elástico, las ecuaciones que definen el sistema pueden expresarse de forma matricial como:

$$
[\mathrm{K}]\{\delta\}=\{\mathrm{F}\}
$$

donde $[\mathrm{K}]$ es la matriz rigidez del sistema, $\{\delta\}$ es el vector 
de desplazamientos y $\{\mathrm{F}\}$ es el vector de fuerzas. Para modelar los elementos de resorte, se utilizaron elementos lineales en 1-D. Los tipos de elementos utilizados en 1-D a través de MEF son: resorte (utilizados en este trabajo), barras y vigas. En ABAQUS se utiliza la conexión axial para simular la unión entre 2 materiales por intermedio de resortes. En este comando se deben incorporar los datos obtenidos del ensayo push-out con el fin de describir el comportamiento del elemento tipo rompecabezas. En una conexión axial entre dos nodos el desplazamiento relativo ocurre a lo largo de la línea que separa a ambos nodos (Figura 16).

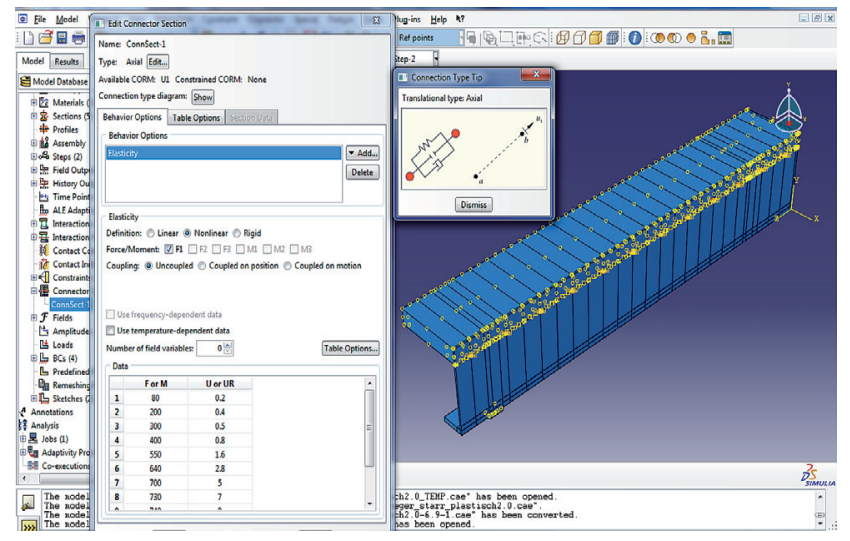

Figura 16: Conexión axial

\section{Conexiones de corte}

En la transferencia de la fuerza cortante y el deslizamiento asociado de la conexión de corte, es importante distinguir entre conexiones rígidas y flexibles. En la Figura 17a se muestra una conexión del tipo rígida, lo que induce una combinación de viga de acero y losa de hormigón sin desplazamientos relativos. Por otra parte, en la Figura $17 \mathrm{~b}$ se muestra una conexión flexible y se puede apreciar que sí existen desplazamientos relativos. Para todos los conectores de corte en la condición de carga se desarrolla un desplazamiento. A partir de los resultados de los ensayos push-out puede ser encontrada la capacidad de carga de los conectores a corte.
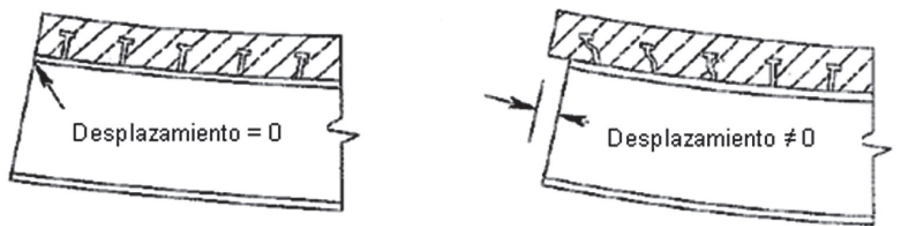

Figura 17: a) Conexión rígida y b) flexible

\section{Análisis de los resultados} Interconexión flexible

La Figura 18 muestra las curvas momento-deflexión obtenidas con el programa ABAQUS con resorte y modelo plástico del material y su comparación con los resultados experimentales. La curva de la simulación está por debajo de la curva obtenida experimentalmente, pero logra predecir la falla con bastante aproximación. Se puede apraciar que hasta una deflexión cercanna a los $13 \mathrm{~mm}$, la simulación indica momentos casi idénticos a los obtenidos en el laboratorio. El porcentaje de error de la simulación numérica es de un 1,6\% aproximadamente respecto al momento último $\mathrm{M}_{\mathrm{u}}$ obtenido del ensayo de laboratorio. El rango lineal-elástico de la simulación muestra una buena aproximación a la viga mixta real.

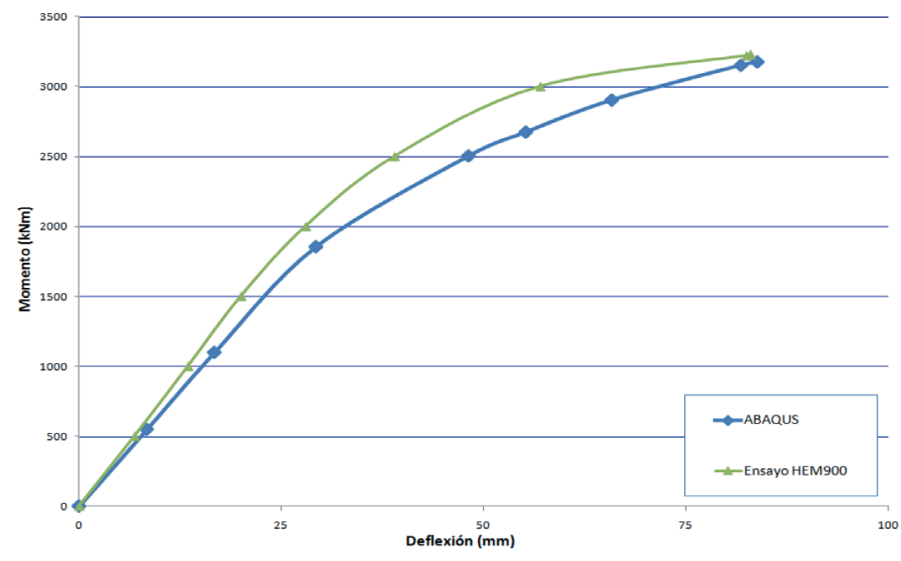

Figura 18: Comparación entre resultados numéricos y de laboratorio de deflexión en la mitad de la viga

Ambas curvas llegan a un punto de convergencia similar. Las diferencias de las curvas se pueden atribuir a la fricción entre el hormigón y el acero. Los resultados de la simulación predicen claramente la curva momentodeflexión obtenidas en el laboratorio, por ende es posible validar el modelo. La Figura 19 muestra la ubicación del strain gauge y la Figura 20 muestra las deflexiones obtenidas de la simulación.

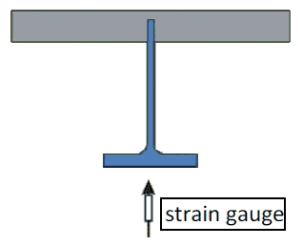

Figura 19: Ubicación del strain gauge 


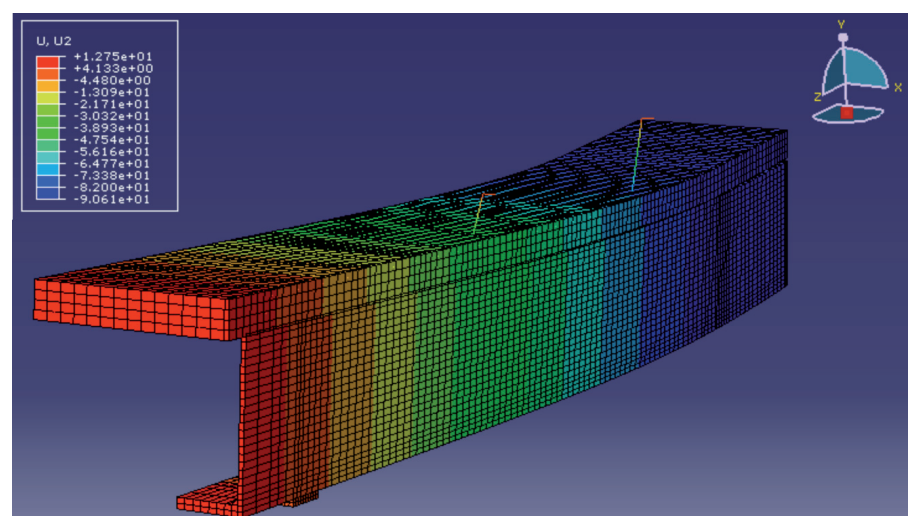

Figura 20: Deformada $u$ en el eje vertical en mm

\section{Interconexión rígida}

La Figura 21 muestra la curva momento-deflexión en el centro de la viga (mayor deflexión) y la Figura 22 muestra la deformada en el eje vertical. Se puede apreciar en la Figura 21 que los resultados no son similares. La diferencia entre el modelo computacional y el ensayo real es de 1000 $\mathrm{kNm}$, lo cual representa un error del $25 \%$ aproximadamente. Por ende se puede concluir que modelar una viga mixta utilizando una unión rígida entre una losa de hormigón y una viga de acero no es una opción válida, ya que no predice el comportamiento del sistema adecuadamente.

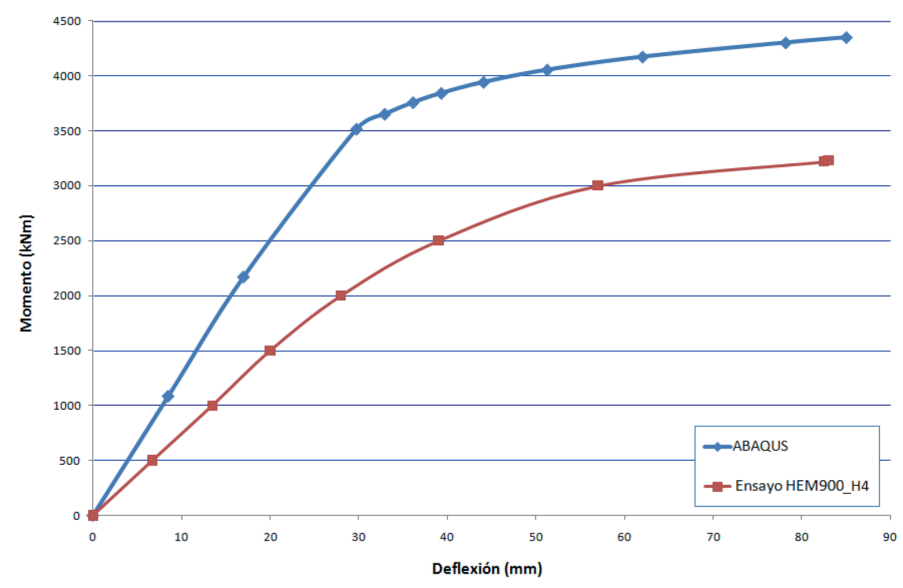

Figura 21: Curva momento - deflexión

\section{Conclusiones}

En el presente trabajo se investiga la interconexión de corte de vigas compuestas de hormigón de ultra alto desempeño UHPC (Ultra High Performance Concrete) y acero de alta resistencia utilizando métodos numéricos. Se modeló con el programa computacional ABAQUS, el cual utiliza elementos finitos tridimensionales, a fin de estudiar la conexión de corte. Para la transferencia de fuerza de corte

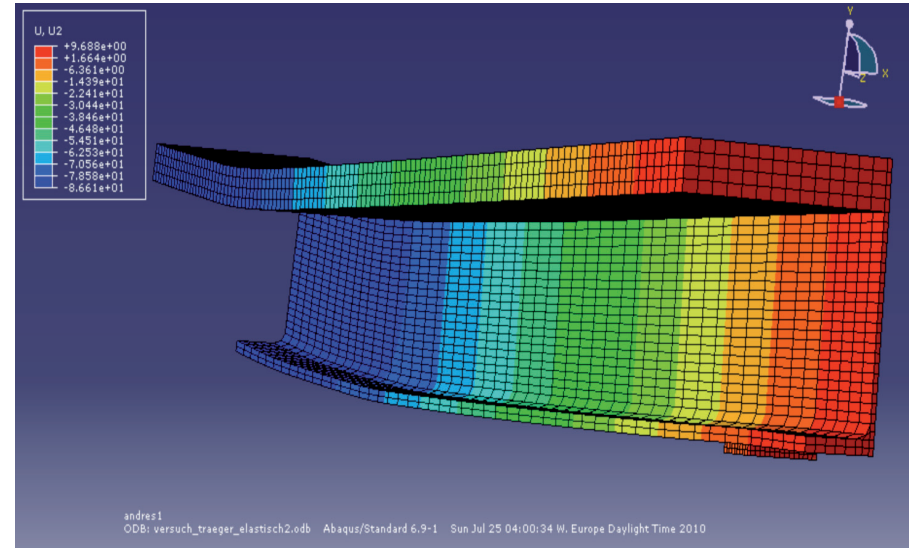

Figura 22: Deformada $u$ en el eje vertical en mm

entre la losa de hormigón y la viga de acero se desarrolló un nuevo tipo de unión, la barra de rompecabezas en forma de puzzle.

Para investigar las fuerzas de corte mediante métodos numéricos existen dos enfoques. Uno con una unión por medio de elementos de resorte no lineal y otro utilizando elementos de interfaz. En el marco de este trabajo se ha centrado en elementos de resorte no lineales.

En primer lugar, el comportamiento no lineal del hormigón UHPC tuvo que ser calibrado. Para ello, se simuló un ensayo a flexotracción para representar correctamente el daño del hormigón en la zona de tensión. Mediante la variación de la energía de rotura así como la resistencia a la tracción se pudo representar el ensayo satisfactoriamente. Lo mismo se hizo con el área de compresión de las pruebas de compresión. Una vez calibrado el modelo numérico se implementó para la sección de la estructura mixta. Esto sirvió para reducir la cantidad de ensayos experimentales a ejecutar, ya que con las simulaciones numéricas se pudo determinar los tipos de conectores más convenientes y así poder realizar menos ensayos experimentales.

Tomando en consideración la similitud entre los resultados numéricos y experimentales, con un porcentaje de error menor al $5 \%$ del $\mathrm{M}_{\mathrm{u}}$, se puede concluir que las hipótesis adoptadas en el modelo numérico son acertadas, lo que da la posibilidad que el fenómeno físico del problema pueda ser reproducido y, en particular, el deslizamiento longitudinal de la interconexión entre la viga de acero y la losa de hormigón. Se confirma la complementariedad 
entre la simulación numérica y el uso de modelos físicos, pues por una parte los ensayos permiten calibrar y validar los modelos a partir de sus respuestas físicas y cuantitativas, mientras que la simulación permite visualizar fenómenos tensionales y deformaciones que no pueden ser cuantificados adecuadamente con los métodos experimentales.

\section{Agradecimientos}

Se agradece al Servicio de Intercambio Académico Alemán DAAD y a la Universidad Católica de la Santísima Concepción por las becas que permitieron realizar esta investigación. Se agradece especialmente al Dipl.-Ing. Joerg Gallwoszus por el apoyo otorgado en esta investigación.

\section{Referencias}

ABAQUS (2009). Online documentation, Version 6.9. Hibitt, Karlsson \& Sorensen

Aribert, J.M. and Labib, A.G. (1982). Modèle de calcul élastoplastique de poutres mixtes à connexion partielle. Construction Métallique 4: 3-52

Eurocode 4 (2004). Bemessung und Konstruktion von Verbundtragwerken aus Stahl und Beton. Teil 1-1: Allgemeine Bemessungsregeln und Anwendungsregeln für den Hochbau.

Kaufmann, M. (2010). Längsschubtragfähigkeit von Verbundträgern aus UHPC und hochfestem Stahl unter positiver Momentenbeanspruchung. Diplomarbeit RWTH- Aachen

Lee, J. and Fenves, G. L. (1998). Plastic-damage model for cyclic loading of concrete structures. Journal of Engineering Mechanics 124(8), 892-900

Rauscher, S., Gallwoszus, J. and Hegger, J. (2011). Innovative shear connectors in UHPC - static loading. Proceedings of Eurosteel $2011-6^{\text {th }}$ European Conference on Steel and Composite Structures, Budapest/Hungary, 483-488.

Teworte, F. (2008). Numerische Untersuchungen zur Abgrenzung zwischen starrer und biegeweicher Lagerung von SpannbetonFertigdecken. Diplomarbeit RWTH-Aachen 